\title{
RESTRICTION OF STABLE BUNDLES IN CHARACTERISTIC $p$
}

\author{
TOHRU NAKASHIMA
}

\begin{abstract}
Let $k$ be an algebraically closed field of characteristic $p>0$. Let $X$ be a nonsingular projective variety defined over $k$ and $H$ an ample line bundle on $X$. We shall prove that there exists an explicit number $m_{0}$ such that if $E$ is a $\mu$-stable vector bundle of rank at most three, then the restriction $E_{\mid D}$ is $\mu$-stable for all $m \geq m_{0}$ and all smooth irreducible divisors $D \in|m H|$. This result has implications to the geometry of the moduli space of $\mu$-stable bundles on a surface or a projective space.
\end{abstract}

\section{INTRODUCTION}

Let $X$ be a nonsingular projective variety of dimension $n$ defined over an algebraically closed field $k$, and let $E$ be a vector bundle of rank $r$ on $X$. The problem of whether the $\mu$-(semi)stability of $E$ with respect to an ample divisor $H$ is preserved under restriction to a divisor $D \in|m H|$ has been studied intensively in recent years. When the characteristic of $k$ is arbitrary, a fundamental theorem of Mehta-Ramanathan states that the answer is positive for a general member $D$ and sufficiently large $m$ ([M-R1], [M-R2]). However, most of the important results have been proved under the assumption that the characteristic is zero. For example, $\mathrm{H}$. Flenner's theorem on the effective estimate of $m$ has no known analogue in positive characteristic $([\mathrm{F}])$.

In this paper we would like to contribute to the restriction problem when char $k=$ $p>0$. Under the assumption that $r=2$ or 3 , we shall give an explicit number $m_{0}$ such that for all $m \geq m_{0}$, every $\mu$-stable bundle $E$ with fixed Chern classes remains $\mu$-stable when restricted to arbitrary smooth irreducible divisors $D \in|m H|$. As a consequence, we obtain the restriction morphism between moduli spaces of $\mu$-stable bundles

$$
j_{D}: M_{X}\left(r, c_{i}\right) \rightarrow M_{D}\left(r, c_{i \mid D}\right),
$$

where $M_{X}\left(r, c_{i}\right)$ denotes the moduli space of $\mu$-stable bundles $E$ of rank $r(r=2,3)$ with Chern classes $c_{i}(E)=c_{i}$. We remark that a restriction theorem with no genericity assumption on $D$ has been obtained by $\mathrm{F}$. Bogomolov if $n=2$ and char $k=0$ ([B1], [B2]). Recently, A. Moriwaki has generalized the result to higher dimensional varieties ([Mo3]).

The lower bound $m_{0}$ mentioned above can be expressed as a function of $\delta_{H}(E)$ and $\mu_{H}(X)$, where $\delta_{H}(E)=\left\{2 r c_{2}(E)-(r-1) c_{1}(E)^{2}\right\} \cdot H^{n-2}$ and $\mu_{H}(X)$ denotes the minimal slope of the Harder-Narasimhan filtration of the tangent bundle of $X$. The appearance of the number $\mu_{H}(X)$ comes from our use of an analogue of

Received by the editors April 30, 1996.

1991 Mathematics Subject Classification. Primary 14D20, 14F05.

(C)1997 American Mathematical Society 
Bogomolov-Gieseker inequality, which has been proved for rank two bundles in [N1]. The inequality is described as follows. If $E$ is a $\mu$-semistable torsion-free sheaf of rank $r=2$ or 3 , we have $\delta_{H}(E) \geq 0$ if $\mu_{H}(X) \geq 0$, and otherwise

$$
\delta_{H}(E) \geq-\alpha \frac{\mu_{H}(X)^{2}}{p^{2} H^{n}},
$$

where $\alpha=1$ if $r=2, \alpha=9$ if $r=3$. Inequalities of different types have been obtained for rank two bundles on surfaces ([S-B] ) and non-uniruled varieties ([Mo2]).

It is natural to ask whether the results in the present paper generalize to bundles of rank greater than three. This problem is closely related to the boundedness of the family of $\mu$-semistable sheaves in positive characteristic, which is at present unknown except when $r \leq 3$ or $n=2$. Roughly speaking, the restriction theorem, the Bogomolov-Gieseker inequality and the boundedness may be considered as almost equivalent properties of $\mu$-semistable sheaves.

In the first section we will prove analogues of the Bogomolov-Gieseker inequality. The restriction theorem will be proved in section two. This result will be applied in the last section to the study of moduli spaces of $\mu$-stable bundles on surfaces and projective spaces.

The author is very grateful to the referee for pointing out several inaccuracies in the original version of this paper.

\section{The Bogomolov-Gieseker Inequality}

Throughout this paper all varieties are defined over an algebraically closed field $k$ of characteristic $p>0$. Let $X$ be a nonsingular projective variety of dimension $n \geq 2$ over $k$ and $E$ a rank $r$ torsion-free sheaf on $X$. For an ample line bundle $H$ on $X$, we define the slope of $E$ by

$$
\mu_{H}(E)=\frac{c_{1}(E) \cdot H^{n-1}}{r}
$$

and let

$$
\delta_{H}(E):=\left\{2 r c_{2}(E)-(r-1) c_{1}(E)^{2}\right\} \cdot H^{n-2} .
$$

Even if $E$ is $\mu$-semistable, the Bogomolov-Gieseker inequality $\delta_{H}(E) \geq 0$ does not hold in general. Nevertheless, one expects that some lower bound for $\delta_{H}(E)$, which depends on $X$ and $H$, may exist. In fact, the boundedness of semistable sheaves implies the existence of a lower bound. For rank two $\mu$-semistable bundles, an effective lower bound has been given in [N1]. The purpose of this section is to extend the result to $\mu$-semistable torsion-free sheaves of rank at most three.

A torsion-free sheaf $E$ is said to be p-semistable with respect to $H$ if, for all $m \geq 0$, the $m$-th iterated Frobenius pull-back $\left(F^{m}\right)^{*} E$ is $\mu$-semistable with respect to $H$. The following result has been proved for vector bundles in [Mo1, Theorem $1]$, and its generalization to torsion-free sheaves poses no significant problems.

Proposition (1.1). Let $X$ be a nonsingular projective variety of dimension $n \geq 2$. Let $E$ be a torsion-free sheaf of rank $r$ on $X$ which is p-semistable with respect to $H$. Then $\delta_{H}(E) \geq 0$ if $r \leq 3$.

Let $T_{X}$ denote the tangent bundle of $X$ and let

$$
0=T_{0} \subset T_{1} \subset \cdots \subset T_{s-1} \subset T_{s}=T_{X}
$$


be the Harder-Narasimhan filtration (H-N filtration for short) with respect to $H$. We define

$$
\mu_{H}(X):=\mu_{H}\left(T_{X} / T_{s-1}\right) .
$$

Proposition (1.2). Assume that $\mu_{H}(X) \geq 0$, and let $E$ be a $\mu$-semistable torsionfree sheaf of rank $r$. Then $\delta_{H}(E) \geq 0$ if $r \leq 3$.

Proof. It suffices to show that under the assumption $\mu_{H}(X) \geq 0$, every $\mu$-semistable sheaf $E$ is $p$-semistable. Assume that $E$ is not $p$-semistable, and let $m$ be the smallest integer such that $\left(F^{m}\right)^{*} E$ is unstable. Let

$$
0=E_{0} \subset E_{1} \subset \cdots \subset E_{s-1} \subset E_{s}=\left(F^{m}\right)^{*} E
$$

be the H-N filtration of $\left(F^{m}\right)^{*} E$ and let $G_{i}=E_{i} / E_{i-1}$. By arguing as in the proof of [N1, Lemma 1], for $0<i<s$ we obtain a non-trivial $\mathcal{O}_{X}$-homomorphism

$$
f_{i}: T_{X} \rightarrow \mathcal{H o m}_{\mathcal{O}_{X}}\left(E_{1},\left(F^{m}\right)^{*} E / E_{1}\right) .
$$

It follows that we can find $0<i<j \leq s$ and a non-zero map

$$
f: T_{X} \rightarrow \mathcal{H} m_{\mathcal{O}_{X}}\left(G_{i}, G_{j}\right) .
$$

Since at least one of $G_{i}$ and $G_{j}$ is of rank one, the latter sheaf is $\mu$-semistable. Hence we obtain $\mu_{H}(X) \leq \mu_{H}\left(G_{j}\right)-\mu_{H}\left(G_{i}\right)<0$, which is a contradiction. This completes the proof.

Remark. The condition $\mu_{H}(X) \geq 0$ is satisfied for varieties with nef tangent bundle $T_{X}$ (that is, the tautological bundle $\mathcal{O}(1)$ on $\mathbb{P}\left(T_{X}\right)$ is nef). Another example is a Fano $n$-fold $X$ such that $T_{X}$ is $\mu$-semistable with respect to $H=-K_{X}$ : we have $\mu_{H}(X)=\left(-K_{X}\right)^{n} / n>0$ in this case. If $X$ is a del Pezzo surface, the $\mu$-semistability of $T_{X}$ has been proved ([Fa]).

Corollary (1.3). Let $X$ be a Fano threefold. If the tangent bundle of $X$ is $\mu$ semistable with respect to $-K_{X}$, then $c_{1}(X)^{3} \leq 72$.

Proof. Since we have $\mu_{H}(X)=\mu_{H}\left(T_{X}\right)=\left(-K_{X}\right)^{3} / 3>0$, Proposition (1.2) yields

$$
\left\{3 c_{2}(X)-c_{1}(X)^{2}\right\} \cdot c_{1}(X) \geq 0 .
$$

We have $H^{3}\left(X, \mathcal{O}_{X}\right) \cong H^{0}\left(X, K_{X}\right)=0$. Further, by [N1, Corollary 3]

$$
H^{2}\left(X, \mathcal{O}_{X}\right) \cong H^{1}\left(X, K_{X}\right)=0 \text {. }
$$

Hence $\chi\left(\mathcal{O}_{X}\right)=1-h^{1}\left(\mathcal{O}_{X}\right) \leq 1$ and, by the Riemann-Roch formula,

$$
1 \geq \frac{1}{24} c_{1}(X) c_{2}(X) \text {. }
$$

Therefore we obtain $c_{1}(X)^{3} \leq 72$, as desired.

In view of Proposition (1.1), the following can be proved as in [N1, Theorem 1].

Proposition (1.4). Let $X$ be a nonsingular projective variety of dimension $n \geq 2$. Let $E$ be a rank two torsion-free sheaf which is $\mu$-semistable with respect to $H$. Then

1. If $\mu_{H}(X) \geq 0$, then $\delta_{H}(E) \geq 0$.

2. If $\mu_{H}(X)<0$, then

$$
\delta_{H}(E) \geq-\frac{\mu_{H}(X)^{2}}{p^{2} H^{n}}
$$


The rest of this section is devoted to proving an inequality of the above type for rank three $\mu$-semistable sheaves. The following lemma is elementary but very useful.

Lemma (1.5). Let $E$ be a torsion-free sheaf of rank $r$ on $X$. Assume that $E$ has a filtration

$$
0=E_{0} \subset E_{1} \subset \cdots \subset E_{s-1} \subset E_{s}=E
$$

such that $G_{i}=E_{i} / E_{i-1}$ is torsion-free of rank $r_{i}$ for each $i$. We put

$$
\alpha_{i}=\frac{r\left(\mu_{H}\left(G_{i}\right)-\mu_{H}(E)\right)}{H^{n}}
$$

Then

$$
\delta_{H}(E) \geq \sum_{i=1}^{s}\left\{\frac{r}{r_{i}} \delta_{H}\left(G_{i}\right)-\frac{r_{i}}{r} \alpha_{i}^{2} H^{n}\right\}
$$

Proof. We have

$$
\begin{aligned}
2 c_{2}(E) \cdot H^{n-2} & =\left\{2 \sum_{i=1}^{s} c_{2}\left(G_{i}\right)+2 \sum_{i<j} c_{1}\left(G_{i}\right) c_{1}\left(G_{j}\right)\right\} \cdot H^{n-2} \\
& =\left\{2 \sum_{i=1}^{s} c_{2}\left(G_{i}\right)+c_{1}(E)^{2}-\sum_{i=1}^{s} c_{1}\left(G_{i}\right)^{2}\right\} \cdot H^{n-2} \\
& \geq \sum_{i=1}^{s}\left(\frac{\delta_{H}\left(G_{i}\right)}{r_{i}}-\frac{c_{1}\left(G_{i}\right)^{2} \cdot H^{n-2}}{r_{i}}\right)+c_{1}(E)^{2} \cdot H^{n-2} .
\end{aligned}
$$

On the other hand, since for each $i$ we have

$$
\left\{\frac{r}{r_{i}} c_{1}\left(G_{i}\right)-c_{1}(E)-\alpha_{i} H\right\} \cdot H^{n-1}=0
$$

the Hodge index theorem yields

$$
\left\{\frac{r}{r_{i}} c_{1}\left(G_{i}\right)-c_{1}(E)-\alpha_{i} H\right\}^{2} \cdot H^{n-2} \leq 0
$$

Hence

$$
-\frac{c_{1}\left(G_{i}\right)^{2} \cdot H^{n-2}}{r_{i}} \geq\left\{-\frac{2}{r} c_{1}\left(G_{i}\right) \cdot c_{1}(E)+\frac{r_{i}}{r^{2}} c_{1}(E)^{2}\right\} \cdot H^{n-2}-\frac{r_{i}}{r^{2}} \alpha_{i}^{2} H^{n} .
$$

Since $\sum_{i=1}^{s} c_{1}\left(G_{i}\right)=c_{1}(E)$ and $\sum_{i=1}^{s} r_{i}=r$, after summing up these inequalities for $1 \leq i \leq s$, we obtain

$$
\sum_{i=1}^{s}-\frac{c_{1}\left(G_{i}\right)^{2} \cdot H^{n-2}}{r_{i}} \geq-\frac{c_{1}(E)^{2} \cdot H^{n-2}}{r}-\sum_{i=1}^{s} \frac{r_{i}}{r^{2}} \alpha_{i}^{2} H^{n} .
$$

Combining this with the inequality for $2 c_{2}(E) \cdot H^{n-2}$, we obtain the claimed bound for $\delta_{H}(E)$. 
Assume that $\mu_{H}(X)<0$. Let $E$ be a $\mu$-semistable torsion-free sheaf of rank three such that $F^{*} E$ is not $\mu$-semistable. Let

$$
0=E_{0} \subset E_{1} \subset \cdots \subset E_{s-1} \subset E_{s}=F^{*} E
$$

be the H-N filtration $(s=2$ or 3$)$. Let $G_{i}=E_{i} / E_{i-1}$ and $r_{i}=\operatorname{rk} G_{i}$. There are three types of $\left(r_{i}\right):(2,1),(1,2),(1,1,1)$. We have the following estimates for $\alpha_{i}$.

Lemma (1.6). $\alpha_{1}>0$, and

$$
\begin{aligned}
\frac{2 \mu_{H}(X)}{H^{n}} & \leq \alpha_{2}<\alpha_{1} \leq-\frac{\mu_{H}(X)}{H^{n}} \quad \text { in case of type }(2,1), \\
\frac{\mu_{H}(X)}{H^{n}} & \leq \alpha_{2}<\alpha_{1} \leq-\frac{2 \mu_{H}(X)}{H^{n}} \quad \text { in case of type }(1,2), \\
\frac{3 \mu_{H}(X)}{H^{n}} & \leq \alpha_{3}<\alpha_{2}<\alpha_{1} \leq-\frac{3 \mu_{H}(X)}{H^{n}} \quad \text { in case of type }(1,1,1) .
\end{aligned}
$$

Proof. The inequality $\alpha_{1}>0$ is clear. As in the proof of Proposition (1.2), for each $i=1,2$, we have a non-trivial $\mathcal{O}_{X}$-homomorphism

$$
f_{i}: T_{X} \rightarrow \mathcal{H o m}_{\mathcal{O}_{X}}\left(E_{i}, F^{*} E / E_{i}\right)=: H_{i}
$$

Since the slope of the maximal destabilizing subsheaf of $H_{i}$ is $\mu_{H}\left(G_{i+1}\right)-\mu_{H}\left(G_{i}\right)$, we see that $\mu_{H}(X) \leq \mu_{H}\left(G_{i+1}\right)-\mu_{H}\left(G_{i}\right)$ for $i=1,2$. Then it is easy to deduce the following estimates for $\mu_{H}\left(E_{1}\right)$ :

$$
\begin{aligned}
& \mu_{H}\left(E_{1}\right) \leq \mu_{H}\left(F^{*} E\right)-\frac{2}{3} \mu_{H}(X) \quad \text { in case of type }(2,1), \\
& \mu_{H}\left(E_{1}\right) \leq \mu_{H}\left(F^{*} E\right)-\frac{1}{3} \mu_{H}(X) \quad \text { in case of type }(1,2), \\
& \mu_{H}\left(E_{1}\right) \leq \mu_{H}\left(F^{*} E\right)-\mu_{H}(X) \quad \text { in case of type }(1,1,1) .
\end{aligned}
$$

The claim follows immediately from the above inequalities.

Theorem (1.7). Let $X$ be a nonsingular projective variety of dimension $n \geq 2$ with an ample line bundle $H$. Let $E$ be a $\mu$-semistable torsion-free sheaf of rank three on $X$.

1. If $\mu_{H}(X) \geq 0$, then $\delta_{H}(E) \geq 0$.

2. If $\mu_{H}(X)<0$, then

$$
\delta_{H}(E)>-\frac{9 \mu_{H}(X)^{2}}{p^{2} H^{n}} .
$$

Proof. (1) follows from Proposition (1.2). Assume $\mu_{H}(X)<0$. Let $m$ denote the smallest integer such that $\left(F^{m}\right)^{*} E$ is not $\mu$-semistable. Let

$$
0=E_{0} \subset E_{1} \subset \cdots \subset E_{s-1} \subset E_{s}=\left(F^{m}\right)^{*} E
$$

be the H-N filtration of $\left(F^{m}\right)^{*} E$ and put $G_{i}=E_{i} / E_{i-1}$. We will give the lower bounds for $\delta_{H}(E)$ according to the three types of $r_{i}=\operatorname{rk} G_{i}$. In case of type $(2,1)$, we have $\delta_{H}\left(G_{1}\right) \geq-\mu_{H}(X)^{2} /\left(p^{2} H^{n}\right)$ by Proposition (1.4) and $\delta_{H}\left(G_{2}\right) \geq 0$. Applying Lemmas (1.5) and (1.6) to $\left(F^{m-1}\right)^{*} E$, we obtain

$$
\delta_{H}\left(\left(F^{m}\right)^{*} E\right)=p^{2 m} \delta_{H}(E)>-\left(2+\frac{3}{2 p^{2}}\right) \frac{\mu_{H}(X)^{2}}{H^{n}} .
$$


Hence

$$
\delta_{H}(E)>-\left(2+\frac{3}{2 p^{2}}\right) \frac{\mu_{H}(X)^{2}}{p^{2} H^{n}} .
$$

Similarly, in case of type $(1,2)$

$$
\delta_{H}(E)>-\left(4+\frac{3}{2 p^{2}}\right) \frac{\mu_{H}(X)^{2}}{p^{2} H^{n}} .
$$

Finally, in case of type $(1,1,1)$ we obtain

$$
\delta_{H}(E)>-\frac{9 \mu_{H}(X)^{2}}{p^{2} H^{n}} .
$$

Comparing the above bounds for $\delta_{H}(E)$, we get the theorem.

Corollary (1.8). Assume that $X$ is a threefold with the ample canonical bundle $K_{X}$. If the tangent bundle of $X$ is $\mu$-semistable with respect to $K_{X}$, then the following inequality holds:

$$
\left\{3 c_{2}(X)-c_{1}(X)^{2}\right\} \cdot\left(-c_{1}(X)\right) \geq 0 .
$$

Proof. Applying Theorem (1.7) to $E=T_{X}$ and $H=K_{X}$, we obtain

$$
\left\{3 c_{2}(X)-c_{1}(X)^{2}\right\} \cdot\left(-c_{1}(X)\right)>-\frac{3}{2 p^{2}}>-1 .
$$

The claim is clear, since the left-hand side is an integer.

\section{THE RESTRICTION THEOREM}

Let $X$ be a nonsingular projective variety of dimension $n \geq 2$ and let $H$ be an ample line bundle on $X$. In this section we will prove the restriction theorem mentioned in the introduction. We will treat the two cases $r=2$ and $r=3$ separately. Let $\delta=\delta_{H}(E), \mu=\mu_{H}(X)$ and $h=H^{n}$.

Theorem (2.1). Let $E$ be a rank two vector bundle on $X$ which is $\mu$-stable with respect to $H$. Let $D \subset X$ be a smooth irreducible divisor with $D \in|m H|$. Then $E_{\mid D}$ is $\mu$-stable with respect to $H_{\mid D}$ under either one of the following conditions:

1. $\mu \geq 0$ and $m>\frac{1}{2}\left(\delta+\frac{1}{h}\right)$;

2. $\mu<0$ and

$$
m>\max \left\{\sqrt{\frac{1}{h}\left(\delta+\frac{\mu^{2}}{p^{2} h}\right)}, \frac{1}{2}\left(\delta+\frac{1}{h}\right)\right\} .
$$

Proof. Assume that $E_{\mid D}$ is not $\mu$-stable with respect to $H_{\mid D}$. Then there exist a rank one torsion-free sheaf $Q$ on $D$ satisfying $\mu_{H_{\mid D}}(Q) \leq \mu_{H_{\mid D}}\left(E_{\mid D}\right)$ and a surjection $E_{\mid D} \rightarrow Q$. Let $E^{\prime}$ be the rank two torsion-free sheaf on $X$ defined by the exact sequence

$$
0 \rightarrow E^{\prime} \rightarrow E \rightarrow Q \rightarrow 0
$$

Then we have $c_{1}\left(E^{\prime}\right)=c_{1}(E)-D$ and $c_{2}\left(E^{\prime}\right)=c_{2}(E)-c_{1}(E) \cdot D+c_{1}(Q) \cdot H_{\mid D}$. Hence

$$
\delta_{H}\left(E^{\prime}\right) \leq \delta_{H}(E)-m^{2} H^{n}
$$


By Proposition (1.4), it follows that under assumption (1) or (2), $E^{\prime}$ is unstable with respect to $H$. Consider the $\mathrm{H}-\mathrm{N}$ filtration of $E^{\prime}$ :

$$
0 \rightarrow G_{1} \rightarrow E^{\prime} \rightarrow G_{2} \rightarrow 0,
$$

where the $G_{i}$ are rank one torsion-free sheaves. For $i=1$, 2, let

$$
\alpha_{i}=\frac{2\left(\mu_{H}\left(G_{i}\right)-\mu_{H}\left(E^{\prime}\right)\right)}{H^{n}} .
$$

Since $G_{1}$ is a subsheaf of $E$ which is $\mu$-stable, we have

$$
0<\alpha_{1} \leq m-\frac{1}{H^{n}}
$$

and we have $\alpha_{2}=-\alpha_{1}$. Applying Lemma (1.5) to $E^{\prime}$, we have $\delta_{H}\left(E^{\prime}\right) \geq-\alpha_{1}^{2} H^{n} \geq$ $-\left(m-1 /\left(H^{n}\right)\right)^{2} H^{n}$. It follows that

$$
m \leq \frac{1}{2}\left(\delta_{H}(E)+\frac{1}{H^{n}}\right) .
$$

This contradicts the assumption on $m$. Hence the theorem is proved.

Theorem (2.2). Let $X, H$ be as before and let $E$ be a rank three vector bundle on $X$ which is $\mu$-stable with respect to $H$. Then for every smooth irreducible $D \in|m H|$, $E_{\mid D}$ is $\mu$-stable under one of the following conditions:

1. $\mu \geq 0$ and

$$
m>\max \left\{\sqrt{\frac{\delta}{2 h}}, \sqrt{\frac{2}{3 h}\left(\delta+\frac{2}{h}\right)}, \frac{1}{12}\left(\delta+\frac{1}{2 h}\right), \frac{1}{2}\left(\frac{\delta}{2}+\frac{1}{h}\right)\right\} ;
$$

2. $\mu<0$ and

$$
\begin{aligned}
m>\max \left\{\sqrt{\frac{1}{2 h}\left(\delta+\frac{9 \mu^{2}}{p^{2} h}\right)}, \sqrt{\frac{2}{3 h}\left(\delta+\frac{2}{h}+\frac{\mu^{2}}{p^{2} h}\right)},\right. \\
\left.\frac{1}{4}\left(\frac{\delta}{3}+\frac{\mu^{2}}{2 p^{2} h}\right), \frac{1}{2}\left(\frac{\delta}{2}+\frac{1}{2 h}\right)\right\} .
\end{aligned}
$$

Proof. The proof is similar to the rank two case. Assume that $E_{\mid D}$ is not $\mu$-stable and let $Q$ be its destabilizing torsion-free quotient. First we treat the case when $Q$ is of rank one. Let $E^{\prime}$ be the kernel of the surjection $E_{\mid D} \rightarrow Q$. As before, we have

$$
\delta_{H}\left(E^{\prime}\right) \leq \delta_{H}(E)-2 m^{2} H^{n} .
$$

By Theorem (1.7), $E^{\prime}$ is unstable if $m$ satisfies the inequality

$$
m>\max \left\{\sqrt{\frac{\delta_{H}(E)}{2 H^{n}}}, \sqrt{\frac{1}{2 H^{n}}\left(\delta_{H}(E)+\frac{9 \mu_{H}(X)^{2}}{p^{2} H^{n}}\right)}\right\} .
$$

We shall give upper bounds for $m$ according to the types of the H-N filtration of $E^{\prime}$. For $1 \leq i \leq 3$, define

$$
\alpha_{i}=\frac{3\left(\mu_{H}\left(G_{i}\right)-\mu_{H}\left(E^{\prime}\right)\right)}{H^{n}}
$$

Assume that we are in case of type $(2,1)$. Then we have

$$
0<\alpha_{1} \leq m-\frac{1}{2 H^{n}}
$$


since $G_{1}$ is a subsheaf of $E$. Using Proposition (1.4) and Lemma (1.5), we obtain

$$
m \leq \begin{cases}\frac{1}{12}\left(\delta_{H}(E)+\frac{1}{2 H^{n}}\right) & \text { if } \mu_{H}(X) \geq 0, \\ \frac{1}{4}\left(\delta_{H}(E)+\frac{\mu_{H}(X)^{2}}{2 p^{2} H^{n}}\right) & \text { if } \mu_{H}(X)<0 .\end{cases}
$$

Similarly, in case of type $(1,2)$ we have $0<\alpha_{1} \leq m-1 /\left(H^{n}\right)$. Hence

$$
m \leq \begin{cases}\sqrt{\frac{2}{3 H^{n}}\left(\delta_{H}(E)+\frac{2}{H^{n}}\right)}-\frac{1}{H^{n}} & \text { if } \mu_{H}(X) \geq 0, \\ \sqrt{\frac{2}{3 H^{n}}\left(\delta_{H}(E)+\frac{2}{H^{n}}+\frac{\mu_{H}(X)^{2}}{p^{2} H^{n}}\right)}-\frac{1}{H^{n}} & \text { if } \mu_{H}(X)<0 .\end{cases}
$$

Assume that we are in case of type $(1,1,1)$. We have $0<\alpha_{1} \leq m-1 /\left(H^{n}\right)$, since

$$
\mu_{H}\left(G_{1}\right) \leq \mu_{H}\left(E^{\prime}\right)+\frac{m H^{n}-1}{3} .
$$

Hence

$$
\begin{aligned}
\mu_{H}\left(G_{2}\right) & =2 \mu_{H}\left(E_{2}\right)-\mu_{H}\left(G_{1}\right) \\
& >2 \mu_{H}\left(E^{\prime}\right)-\left(\mu_{H}\left(E^{\prime}\right)+\frac{m H^{n}-1}{3}\right) \\
& =\mu_{H}\left(E^{\prime}\right)-\frac{m H^{n}-1}{3} .
\end{aligned}
$$

It follows that $\alpha_{2}>-\left(m-1 /\left(H^{n}\right)\right)$. Finally, we have

$$
\begin{aligned}
\mu_{H}\left(G_{3}\right) & =3 \mu_{H}\left(E^{\prime}\right)-\mu_{H}\left(G_{2}\right)-\mu_{H}\left(G_{3}\right) \\
& >3 \mu_{H}\left(E^{\prime}\right)-2\left(\mu_{H}\left(E^{\prime}\right)+\frac{m H^{n}-1}{3}\right) \\
& =\mu_{H}\left(E^{\prime}\right)-\frac{2\left(m H^{n}-1\right)}{3} .
\end{aligned}
$$

Hence we obtain $\alpha_{3}>-2\left(m-1 /\left(H^{n}\right)\right)$. From these bounds for $\alpha_{i}$, we obtain $\alpha_{i}^{2} \leq\left(m-1 /\left(H^{n}\right)\right)^{2}$ for $i=1,2$, and $\alpha_{3}^{2}<4\left(m-1 /\left(H^{n}\right)\right)^{2}$. Thus, by Lemma $(1.5)$,

$$
\delta\left(E^{\prime}\right)>-2\left(m-\frac{1}{H^{n}}\right)^{2} H^{n}
$$

Therefore we obtain

$$
m \leq \frac{1}{2}\left(\frac{\delta_{H}(E)}{2}+\frac{1}{H^{n}}\right) .
$$

Putting these altogether, we conclude that $E_{\mid D}$ has no destabilizing rank one quotient sheaves. If $Q$ is a sheaf of rank two, then let $\widetilde{Q}:=\bigwedge^{2} Q /$ tors denote the quotient of the exterior product $\bigwedge^{2} Q$ by its torsion subsheaf. Then $\widetilde{Q}$ is a rank one torsion-free sheaf, and there is a surjection

$$
\bigwedge^{2} E_{\mid D} \rightarrow \widetilde{Q}
$$

Since we have $\bigwedge^{2} E \cong E^{\vee}\left(c_{1}(E)\right), \bigwedge^{2} E$ is $\mu$-stable and $\delta_{H}\left(\bigwedge^{2} E\right)=\delta_{H}(E)$. Further, it is easy to see that $\widetilde{Q}$ is a destabilizing quotient of $\bigwedge^{2} E_{\mid D}$. Thus we are reduced to the case $Q$ is of rank one. This completes the proof of the theorem. 


\section{Applichtion to the moduli space}

In this section we consider some applications of the restriction theorem to the study of moduli spaces of $\mu$-stable bundles.

Let $S$ be a nonsingular projective surface with an ample line bundle $H$. For $L \in \operatorname{Pic}(S)$, we denote by $M=M_{S}\left(r, L, c_{2}\right)$ the moduli space of vector bundles of rank $r$ on $S$ with $\operatorname{det}(E) \cong L, c_{2}(E)=c_{2}$ which are $\mu$-stable with respect to $H$. $M_{S}\left(r, L, c_{2}\right)$ has a natural compactification $\bar{M}_{S}\left(r, L, c_{2}\right)$, the moduli of Gieseker semistable torsion-free sheaves. We recall that $\bar{M}_{S}\left(r, L, c_{2}\right)$ is constructed as the geometric invariant theory quotient of a certain Quot scheme Quot ${ }^{s s}([\mathrm{G}])$. Let $\pi:$ Quot $^{s s} \rightarrow \bar{M}_{S}\left(r, L, c_{2}\right)$ be the quotient morphism.

We fix a tautological sheaf $\mathcal{F}$ on $S \times$ Quot $^{s s}$. For a smooth curve $C \subset S$ of genus $g(C)$, we define $\mathcal{F}^{C}=\mathcal{F}_{\mid C \times \mathrm{Quot}^{s s}}$. Let $p_{Q}: C \times \mathrm{Quot}^{s s} \rightarrow \mathrm{Quot}^{s s}$ and $p_{C}: C \times$ Quot $^{s s} \rightarrow C$ be the natural projections. The proof of the following lemma is exactly the same as that of [N2, Proposition 1.3], where the result is stated in the case char $k=0$.

Lemma (3.1). Assume that $c_{1}(L) \cdot C=r d$ for some integer $d$, and let $A$ be a line bundle of degree $-d+g(C)-1$ on $C$. Then there exists a line bundle $\operatorname{Det}_{\mathcal{F}}(C)$ on $\bar{M}_{S}\left(r, L, c_{2}\right)$ such that

$$
\pi^{*} \operatorname{Det}_{\mathcal{F}}(C) \cong \operatorname{det}\left(p_{Q}\right) !\left(\mathcal{F}^{C} \otimes p_{C}^{*} A\right)^{\vee} .
$$

Let $\bar{M}_{C}\left(r, L_{\mid C}\right)$ denote the moduli of semistable rank $r$ vector bundles $F$ on $C$ with $\operatorname{det} F \cong L_{\mid C}$. Then we have

Proposition (3.2). If $r=2$ or 3 , then there exists an explicit number $m_{0}$ such that for all $m \geq m_{0}$ and every smooth curve $C \in|r m H|$, we obtain the restriction morphism of moduli spaces

$$
j_{C}: M_{S}\left(r, L, c_{2}\right) \rightarrow \bar{M}_{C}\left(r, L_{\mid C}\right)
$$

which satisfies $\operatorname{Det}_{\mathcal{F}}(C)_{\mid M} \cong j_{C}^{*} \mathcal{L}_{C}$ for an ample line bundle $\mathcal{L}_{C}$ on $\bar{M}_{C}\left(r, L_{\mid C}\right)$. Furthermore, if $m$ is sufficiently large, $j_{C}$ is an injective immersion (namely, an injective morphism with injective differential map).

Proof. We obtain the number $m_{0}$ immediately from Theorems (2.1) and (2.2). The existence of an ample line bundle $\mathcal{L}_{C}$ with the required property follows from the description of $\operatorname{Pic} \bar{M}_{C}\left(r, L_{\mid C}\right)$ given in [D-N]. Thus the first claim is proved.

For the second claim, note that since $M_{S}\left(r, \mathcal{L}, c_{2}\right)$ is of finite type, if $m$ is sufficiently large we have $H^{q}(S, \mathcal{H o m}(E, F)(-C))=0$ for $q \leq 1$ and every $E, F \in M$ by Serre's vanishing theorem. It follows that

$$
H^{0}(S, \mathcal{H o m}(E, F)) \cong H^{0}\left(C, \mathcal{H o m}\left(E_{\mid C}, F_{\mid C}\right)\right)
$$

and the natural map $H^{1}(S, \mathcal{H o m}(E, F)) \rightarrow H^{1}\left(C, \mathcal{H o m}\left(E_{\mid C}, F_{\mid C}\right)\right)$ is injective. Therefore we deduce that $j_{C}$ is an injective immersion.

Next we study $\mu$-stable bundles on the projective space $\mathbb{P}^{n}$ by restricting them to smooth complete intersections. Restrictions to hyperplanes have already been considered in [E] in the case char $k>0$. The following approach to moduli spaces is similar to $[\mathrm{P}]$, which treats the case char $k=0$.

For given integers $r \geq 2$ and $c_{i}(2 \leq i \leq r)$, let $M_{\mathbb{P} n}\left(r, 0, c_{i}\right)$ be the moduli space of rank $r \mu$-stable vector bundle $E$ on $\mathbb{P}^{n}$ with $c_{1}(E)=0$ and $c_{i}(E)=c_{i} \in$ $A^{i}\left(\mathbb{P}^{n}\right) \cong \mathbb{Z}$, where $A^{i}$ denotes the Chow group of codimension $i$ cycles. Similarly, 
for a smooth subvariety $X \subset \mathbb{P}^{n}$, we denote by $M_{X}\left(r, 0, c_{i}\right)$ the moduli space of rank $r$ bundles $E$ on $X$ which are $\mu$-stable with respect to $H=\mathcal{O}_{X}(1)$, with $c_{1}(E)=0, c_{i}(E)=c_{i} H^{i} \in A^{i}(X)$. We notice that if $r \leq 3$, these moduli spaces are quasi-projective schemes ([Ma1], [Ma2]).

Lemma (3.3). Let $X \subset \mathbb{P}^{n}$ be a smooth complete intersection of type $\left(d_{1}, \ldots, d_{m}\right)$ with $\sum_{j=1}^{m} d_{j} \geq n+1$. If $\operatorname{dim}(X) \geq 3$ or $\operatorname{dim}(X)=2$ and $\operatorname{Pic}(X) \cong \mathbb{Z}\left[\mathcal{O}_{X}(1)\right]$, then $T_{X}$ is $\mu$-stable with respect to $\mathcal{O}_{X}(1)$.

Proof. It suffices to prove the $\mu$-stability of the cotangent bundle $\Omega_{X}^{1}$. Assume that $\Omega_{X}^{1}$ is not $\mu$-stable, and let $F \subset \Omega_{X}^{1}$ be a destabilizing subsheaf of rank $r$ with $0<r<n-m$. We may assume $F$ to be reflexive by considering its double dual; hence we obtain an invertible subsheaf $\operatorname{det} F \subset \Omega_{X}^{r}$. Since $\operatorname{Pic}(X) \cong \mathbb{Z}\left[\mathcal{O}_{X}(1)\right]$ if $\operatorname{dim}(X) \geq 3$, we have $\operatorname{det} F=\mathcal{O}_{X}(l)$ for some integer $l$. Thus $H^{0}\left(X, \Omega_{X}^{r}(-l)\right) \neq 0$ and, moreover, the destabilizing condition yields

$$
l \geq \frac{r\left(\sum_{j=1}^{m} d_{j}-n-1\right)}{n-m} \geq 0 .
$$

However, this is a contradiction since we have $H^{0}\left(X, \Omega_{X}^{r}(-l)\right)=0$ for $l \geq 0$ by [D, Théorème 1.5]. This completes the proof.

Theorem (3.4). Let $n \geq 3$, let $c_{i}$ be integers and let $r=2$ or 3 . Let $X \subset \mathbb{P}^{n}$ be a smooth complete intersection of type $\left(d_{1}, \ldots, d_{m}\right)$ with $\operatorname{dim}(X) \geq 2$. There exists an integer $d_{0}$ depending only on $n, m, c_{2}$ and $p$ such that if $d_{j}>d_{0}$, then for all $E \in M_{\mathbb{P}^{n}}\left(r, 0, c_{i}\right), E_{\mid X}$ is $\mu$-stable with respect to $\mathcal{O}_{X}(1)$. Furthermore, for sufficiently large $d_{j}$ and $d=\prod_{j=1}^{m} d_{j}$, the restriction morphism

$$
j_{X}: M_{\mathbb{P} n}\left(r, 0, c_{i}\right) \rightarrow M_{X}\left(r, 0, d c_{i}\right)
$$

defines an open immersion.

Proof. We shall consider only the rank two case, since the proof in the rank three case is entirely similar. First we choose an integer $d_{1}$ such that

$$
d_{1}>\max \left\{2 c_{2}, n+1\right\}
$$

and let $X \subset \mathbb{P}^{n}$ be a smooth hypersurface of degree $d_{1}$. By Theorem (2.1), every $E \in M_{\mathbb{P}^{n}}\left(2,0, c_{2}\right)$ restricts to a $\mu$-stable bundle $E_{\mid X}$ with $c_{1}\left(E_{\mid X}\right)=0, c_{2}\left(E_{\mid X}\right)=$ $c_{2} d_{1} H^{2}$. Since the tangent bundle of $X$ is $\mu$-stable by Lemma (3.3), we have

$$
\mu_{H}(X)=\mu_{H}\left(T_{X}\right)=\frac{\left(n+1-d_{1}\right) d_{1}}{n-1}<0 .
$$

Let $d_{2}$ an integer such that

$$
d_{2}>\max \left\{\sqrt{4 c_{2} d_{1}+\frac{\left(n+1-d_{1}\right)^{2}}{p^{2}(n-1)^{2}}}, 2 c_{2} d_{1}^{2}+\frac{1}{2 d_{1}}\right\} .
$$

Applying again Theorem (2.1) to $E_{\mid X}$, we see that for every smooth $Y \in\left|d_{2} H\right|$, $E_{\mid Y}$ is $\mu$-stable. Repeating this process, we get the first assertion.

Let $\mathcal{I}_{X}$ denote the ideal sheaf of $X$. We observe that if $\operatorname{dim}(X) \geq 2$ and $d_{j}$ are sufficiently large, then we have $H^{q}\left(\mathbb{P}^{n}, \mathcal{H} o m(E, F) \otimes \mathcal{I}_{X}\right)=0$ for $q \leq 2$ and for all $E, F \in M_{\mathbb{P}^{n}}\left(2,0, c_{2}\right)$. Indeed, since $M_{\mathbb{P}^{n}}\left(2,0, c_{2}\right)$ is of finite type, we can use Serre's 
vanishing theorem and the Koszul resolution of $\mathcal{I}_{X}$ to prove the claim. Therefore the cohomology sequences induced by the exact sequence

$$
0 \rightarrow \mathcal{H o m}(E, F) \otimes \mathcal{I}_{X} \rightarrow \mathcal{H o m}(E, F) \rightarrow \mathcal{H o m}\left(E_{\mid X}, F_{\mid X}\right) \rightarrow 0
$$

yield the isomorphisms $H^{q}\left(\mathbb{P}^{n}, \mathcal{H o m}(E, F)\right) \cong H^{q}\left(X, \mathcal{H o m}\left(E_{\mid X}, F_{\mid X}\right)\right)$ for $q \leq 1$. It follows that the restriction morphism defines an open immersion.

For the restriction to complete intersection curves, we have the following result.

Theorem (3.5). Let $n \geq 3, c_{i}$ be integers and $r=2$ or 3 . For sufficiently large $d_{j}$ and a general smooth complete intersection curve $C \subset \mathbb{P}^{n}$ of type $\left(d_{1}, \ldots, d_{n-1}\right)$, every $E \in M_{\mathbb{P}^{n}}\left(r, 0, c_{i}\right)$ restricts to a $\mu$-stable bundle on $C$ and the restriction morphism defines an injective immersion of moduli spaces

$$
j_{C}: M_{\mathbb{P} n}\left(r, 0, c_{i}\right) \rightarrow M_{C}(r, 0) .
$$

Proof. For sufficiently large $d_{j}$, a general complete intersection surface $S \subset \mathbb{P}^{n}$ of type $\left(d_{1}, \ldots, d_{n-2}\right)$ satisfies $\operatorname{Pic}(S) \cong \mathbb{Z}\left[\mathcal{O}_{S}(1)\right]$ (cf. [M-R1, 2.2. Proposition]), and we have $H^{q}\left(\mathbb{P}^{n}, \mathcal{H} \operatorname{om}(E, F) \otimes \mathcal{I}_{C}\right)=0$ for $q \leq 1$. Hence the theorem follows as before.

\section{REFERENCES}

[B1] F.Bogomolov, Holomorphic tensors and vector bundles on projective varieties, Math. of the USSR, Izvestija 13 (1979), 499-555. MR 80j:14014

[B2] F.Bogomolov, Stability of vector bundles on surfaces and curves, Einstein metrics and Yang-Mills connections, Lecture Notes Pure Appl. Math., vol. 145 Marcel Dekker, New York, 1993, 35-49. MR 94i:14021

[D] P.Deligne, Cohomologie des intersections complètes, SGA7, exp.XI., Lecture Notes in Math., 340, Springer, 1973, 39-61. MR 50:7135

[D-N] J.-M.Drezet, M.S.Narasimhan, Groupe de Picard des variétés de modules de fibrés semistables sur les courbe algébriques, Invent.Math. 97 (1989), 53-94. MR 90d:14008

[E] L.Ein, Stable vector bundles on projective spaces in char $p>0$, Math.Ann. 254 (1980), 53-72. MR 81d:14010

[F] H.Flenner, Restriction of semistable bundles on projective varieties, Comment.Math.Helvetici 59 (1984), 635-650. MR 86m:14014

[Fa] R.Fahlaoui, Stabilité du fibré tangent des surfaces de del Pezzo, Math.Ann. 283 (1989), 171-176. MR 89k:14063

[G] D.Gieseker, On the moduli of vector bundles on an algebraic surface, Ann.Math. 106 (1977), 45-60. MR 81h:14014

[Ma1] M.Maruyama, Moduli of stable sheaves I, J.Math. Kyoto Univ. 17 (1977), 91-126. MR $\mathbf{5 6 : 8 5 6 7}$

[Ma2] M.Maruyama, On boundedness of families of torsion free sheaves, J.Math. Kyoto Univ. 21 (1981), 673-701. MR 83a:14019

[Mo1] A.Moriwaki, A note on Bogomolov-Gieseker's inequality in positive characteristic, Duke Math.J 64 (1991), 361-375. MR 92m:14054

[Mo2] A.Moriwaki, Frobenius pull-back of vector bundles of rank 2 over non-uniruled varieties, Math.Ann. 296 (1993), 441-451. MR 94j:14039

[Mo3] A.Moriwaki, Arithmetic Bogomolov-Gieseker's inequality, Amer.J.Math. 117 (1995), 1325-1347. MR 96i: 14022

[M-R1] V.B.Mehta, A.Ramanathan, Semistable sheaves on projective varieties and their restriction to curves, Math.Ann. 258 (1982), 213-224. MR 83f:14013

[M-R2] V.B.Mehta, A.Ramanathan, Restriction of stable sheaves and representations of the fundamental group, Invent. Math. 77 (1984), 163-172. MR 85m:14026

[N1] T.Nakashima, Bogomolov-Gieseker inequality and cohomology vanishing in characteristic p, Proc.Amer.Math.Soc. 123 (1995), 3609-3613. MR 96b:14058

[N2] T.Nakashima, Singularity of the moduli space of stable bundles on surfaces, Compositio Math. 100 (1996), 125-130. MR 97d:14018 
[P] R.Paoletti, Seshadri constants, gonality of space curves, and restriction of stable bundles, J.Diff.Geom. 40 (1994), 475-504. MR 95k:14046

[S-B] N.I.Shepherd-Barron, Unstable vector bundles and linear systems on surfaces in positive characteristic, Invent.Math. 106 (1991), 243-262. MR 92h:14027

Department of Mathematics, Tokyo Metropolitan University, Minami-Ohsawa 1-1, HACHIOJI-SHI, TOKYO, 192-03 JAPAN

E-mail address: nakasima@math.metro-u.ac.jp 